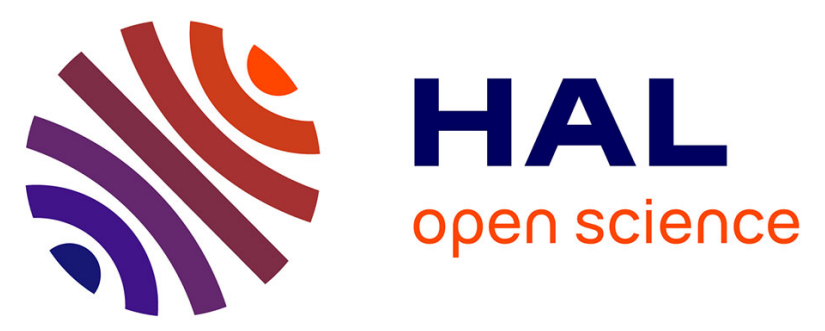

\title{
Evidence of stronger pitch angle scattering loss caused by oblique whistler-mode waves as compared with quasi-parallel waves
}

W Li, D Mourenas, A.V. Artemyev, O. V. Agapitov, J Bortnik, J.M. Albert, R.M. Thorne, B Ni, C. A. Kletzing, W.S. Kurth, et al.

\section{To cite this version:}

W Li, D Mourenas, A.V. Artemyev, O. V. Agapitov, J Bortnik, et al.. Evidence of stronger pitch angle scattering loss caused by oblique whistler-mode waves as compared with quasi-parallel waves. Geophysical Research Letters, 2014, 41, pp.6063-6070. 10.1002/2014GL061260 . insu-01174072

\section{HAL Id: insu-01174072 \\ https://hal-insu.archives-ouvertes.fr/insu-01174072}

Submitted on 8 Jul 2015

HAL is a multi-disciplinary open access archive for the deposit and dissemination of scientific research documents, whether they are published or not. The documents may come from teaching and research institutions in France or abroad, or from public or private research centers.
L'archive ouverte pluridisciplinaire HAL, est destinée au dépôt et à la diffusion de documents scientifiques de niveau recherche, publiés ou non, émanant des établissements d'enseignement et de recherche français ou étrangers, des laboratoires publics ou privés. 


\section{Geophysical Research Letters}

\section{RESEARCH LETTER \\ 10.1002/2014GL061260 \\ Key Points: \\ - Oblique waves cause stronger pitch \\ Evidence of stronger pitch angle scattering loss caused by oblique whistler-mode waves as compared with quasi-parallel waves} angle scattering than parallel waves with the same $B_{\mathrm{w}}$

- We provide the evidence using conjunction events between Radiation Belt Storm Probes (RBSP) and POES

- Both observational and numerical results support this scenario

\author{
Supporting Information: \\ - Readme \\ - Text 01 \\ - Text 02
}

Correspondence to:

W. Li,

moonli@atmos.ucla.edu

\section{Citation:}

Li, W., et al. (2014), Evidence of stronger pitch angle scattering loss caused by oblique whistler-mode waves as compared with quasi-parallel waves, Geophys. Res. Lett., 41, 6063-6070, doi:10.1002/ 2014 GL061260.

Received 18 JUL 2014 Accepted 9 AUG 2014

Accepted article online 12 AUG 2014 Published online 2 SEP 2014 ${ }^{9}$ Department of Physics and Astronomy, University of lowa, lowa City, lowa, USA

\section{Introduction}

\author{
W. Li ${ }^{1}$, D. Mourenas ${ }^{2}$, A. V. Artemyev ${ }^{3,4}$, O. V. Agapitov, ${ }^{5,6}$, J. Bortnik ${ }^{1}$, J. M. Albert ${ }^{7}$, R. M. Thorne', \\ B. $\mathrm{Ni}^{8,1}$, C. A. Kletzing ${ }^{9}$, W. S. Kurth ${ }^{9}$, and G. B. Hospodarsky ${ }^{9}$
}

${ }^{1}$ Department of Atmospheric and Oceanic Sciences, University of California, Los Angeles, Los Angeles, California, USA, ${ }^{2}$ CEA, DAM, DIF, Arpajon, France, ${ }^{3}$ LPC2E/CNRS-University of Orleans, Orleans, France, ${ }^{4}$ Space Research Institute, RAS, Moscow, Russia, ${ }^{5}$ Space Sciences Laboratory, University of California, Berkeley, Berkeley, California, USA, ${ }^{6}$ Taras Shevchenko National University of Kyiv, Kyiv, Ukraine, ${ }^{7}$ Air Force Research Laboratory, Space Vehicles Directorate, Kirtland Air Force Base, New Mexico, USA, ${ }^{8}$ Department of Space Physics, School of Electronic Information, Wuhan University, Wuhan, China,

Abstract Wave normal distributions of lower-band whistler-mode waves observed outside the plasmapause exhibit two peaks: one near the parallel direction and the other at very oblique angles. We analyze a number of conjunction events between the Van Allen Probes near the equatorial plane and Polar Orbiting Environmental Satellites (POES) at conjugate low altitudes, where lower-band whistler-mode wave amplitudes were inferred from the two-directional POES electron measurements over 30-100 keV, assuming that these waves were quasi-parallel. For conjunction events, the wave amplitudes inferred from the POES electron measurements were found to be overestimated as compared with the Van Allen Probes measurements primarily for oblique waves and quasi-parallel waves with small wave amplitudes $(<\sim 20 \mathrm{pT})$ measured at low latitudes. This provides plausible experimental evidence of stronger pitch angle scattering loss caused by oblique waves than by quasi-parallel waves with the same magnetic wave amplitudes, as predicted by numerical calculations.

The wave normal distribution of whistler-mode waves has been a subject of interest over the past few decades based on both satellite observations and ray tracing simulations [e.g., Burton and Holzer, 1974; Goldstein and Tsurutani, 1984; Hayakawa et al., 1984; Li et al., 2011, 2013a; Wilson et al., 2011; Breuillard et al., 2012; Agapitov et al., 2013; Chen et al., 2013; Santolík et al., 2014]. Although wave normal angles of upperband waves (i.e., in the range of $0.5-0.8 f_{\text {ce, }}$ where $f_{\text {ce }}$ is the electron cyclotron frequency) exhibit a broad distribution extending from field-aligned to very oblique angles [Lauben et al., 2002; Hayakawa et al., 1984; Li et al., 2013a], lower-band waves (0.1-0.5 $f_{\text {ce }}$ ) are found to show two predominant peaks in wave normal distribution, one being quasi-parallel and the other very oblique, close to the resonance cone [Santolik et al., 2009; Li et al., 2011, 2013a; Agapitov et al., 2013]. Ray tracing results demonstrated that wave normal angles of whistler-mode waves become more oblique as they propagate from the equator to higher magnetic latitudes [Breuillard et al., 2012; Chen et al., 2013], which is roughly consistent with 10 years of statistical results on whistler-mode wave normal distribution from the Cluster satellites [Agapitov et al., 2013].

Numerical and analytical calculations have shown that electron pitch angle scattering rates driven by very oblique whistler-mode waves are considerably larger at small pitch angles close to the bounce loss cone than scattering by quasi-parallel waves, and thus electron lifetimes can be significantly reduced due to the important contribution of higher-order cyclotron resonances in the energy range under consideration between $\sim 30 \mathrm{keV}$ and $\sim 1 \mathrm{MeV}$ [Artemyev et al., 2012; Mourenas et al., 2012]. The effect of different wave normal distributions of whistler-mode waves on diffusion rates has also been discussed in a few other previous studies [e.g., Shprits and Ni, 2009; Albert, 2012]. Therefore, a realistic treatment of the whistler-mode wave normal distribution is crucial in quantifying energetic electron dynamics in the inner magnetosphere.

Based on the fact that whistler-mode waves play a dominant role in electron precipitation over the energy range of 30-100 keV [Lam et al., 2010; Thorne et al., 2010], Li et al. [2013b] adopted a physics-based technique to infer lower-band wave amplitudes from the ratio of precipitated and trapped electron fluxes measured by 
Polar Orbiting Environmental Satellites (POES) over energies of 30-100 keV. This technique has been validated by analyzing conjunction events between the Van Allen Probes and POES satellites near the conjugate field line at low altitudes and was shown to produce reasonable estimates of whistler-mode wave amplitudes [Li et al., 2013b]. Particularly, the inferred and measured wave amplitudes exhibited remarkable agreement for relatively strong wave amplitudes $\left(B_{\mathrm{w}}>\sim 20 \mathrm{pT}\right)$. However, the inferred wave amplitudes were overestimated compared to the direct wave measurements by the Van Allen Probes at lower wave amplitudes $\left(B_{\mathrm{w}}<\sim 20 \mathrm{pT}\right)$. It is important to note that in this process of inferring whistler-mode wave amplitudes from the two-directional POES electron measurements, we assume that the whistler-mode waves are quasi-parallel. The inconsistency for weak waves might therefore be related to this assumption.

In the present study, by categorizing hundreds of conjunction events between Van Allen Probe wave measurements and POES electron measurements into quasi-parallel or oblique whistler-mode wave events (determined from the Van Allen Probe wave observation), we investigate whether evidence of stronger pitch angle scattering caused by oblique waves (as compared with quasi-parallel waves) can be found from the lowaltitude energetic electron measurements made by the POES satellites. In section 2, we show observational results from the Van Allen Probes and the POES satellites. Numerical calculations were performed to interpret the observational results, which are described in sections 3 and 4. Finally, we summarize and discuss the principal findings of the present study in section 5 .

\section{Observational Results}

The twin Van Allen Probes with an apogee of $\sim 5.8 R_{\mathrm{E}}$ [Mauk et al., 2012] are ideally suited to measure whistlermode waves in the near-equatorial inner magnetosphere. The Electric and Magnetic Field Instrument Suite and Integrated Science (EMFISIS) provides measurements of DC magnetic fields and a comprehensive set of wave electric and magnetic fields [Kletzing et al., 2013]. The Waveform Receiver (WFR) on the EMFISIS Waves instruments not only measures wave power spectral density but also provides wave polarization properties in the survey-mode from $10 \mathrm{~Hz}$ up to $12 \mathrm{kHz}$ with a $6 \mathrm{~s}$ time resolution [Kletzing et al., 2013]. These polarization properties include wave normal angle, ellipticity, planarity, etc. calculated by the Singular Value Decomposition method [Santolik et al., 2003]. The High Frequency Receiver (HFR) is designed to provide electric spectral intensity between 10 and $\sim 400 \mathrm{kHz}$, thus enabling the detection of the upper hybrid resonance frequency, from which the total plasma density can be calculated. This inferred total plasma density is used to determine whether observations are made in the plasmaspheric- or plasmatrough-like region. Specifically, if the plasma density is lower (higher) than the smaller value between $10 \times(6.6 / L)^{4}$ and $30 \mathrm{~cm}^{-3}$, the region is defined to be a plasmatrough (plasmaspheric) region, following Li et al. [2010a]. Only whistler-mode waves observed in the plasmatrough region are analyzed in the present study to exclude plasmaspheric hiss emissions.

The ratios of precipitated $\left(J_{0}\right)$ and trapped electron fluxes $\left(J_{90}\right)$ over 30-100 keV measured by low-altitude POES satellites [Evans and Greer, 2004; Green, 2013] are used to infer whistler-mode wave amplitudes using a physics-based technique described in Li et al. [2013b] and Ni et al. [2014]. Although the detailed description of this technique is not repeated here, it is important to note that in the process of inferring whistler-mode wave amplitudes, we assume that the wave normal angle distribution is quasi-parallel and does not change with magnetic latitude. Finally, we only infer whistler-mode wave amplitudes when both precipitated and trapped electron fluxes are sufficiently larger than the background level $\left(\sim 500 \mathrm{~cm}^{-1} \mathrm{~s}^{-1} \mathrm{sr}^{-1}\right)$, and set the wave amplitude to a very small value (1 pT) otherwise.

Figure 1 shows whistler-mode waves directly measured by both Van Allen Probes and inferred from the twodirectional POES electron measurements. The presence of both lower-band $\left(0.1-0.5 f_{\text {ce }}\right)$ and upper-band waves $\left(0.5-0.8 f_{\text {ce }}\right)$ is clearly visible near apogee along the satellite trajectories. Since 30-100 keV electrons predominantly resonate with lower-band whistler-mode waves [Ni et al., 2008; Li et al., 2010b], we only evaluate wave amplitudes and wave normal angles for lower-band waves in the following analysis. Figures $1 \mathrm{c}$ and if show wave normal angles of lower-band waves where the values are only shown for the data points which satisfy the following four criteria: (1) observed in the plasmatrough region, (2) wave frequencies between 0.1 and $0.5 f_{\text {cer }}$ (3) ellipticity $>0.7$, and (4) planarity $>0.4$. Lower-band wave amplitudes directly measured by the two Van Allen Probes and inferred from multiple POES satellites were both sorted into bins with a size of $0.5 \mathrm{~h}(\mathrm{UT}) \times 0.5 \mathrm{~L} \times 0.5 \mathrm{~h}(\mathrm{MLT})$, and the root mean square (RMS) values of wave amplitudes were 


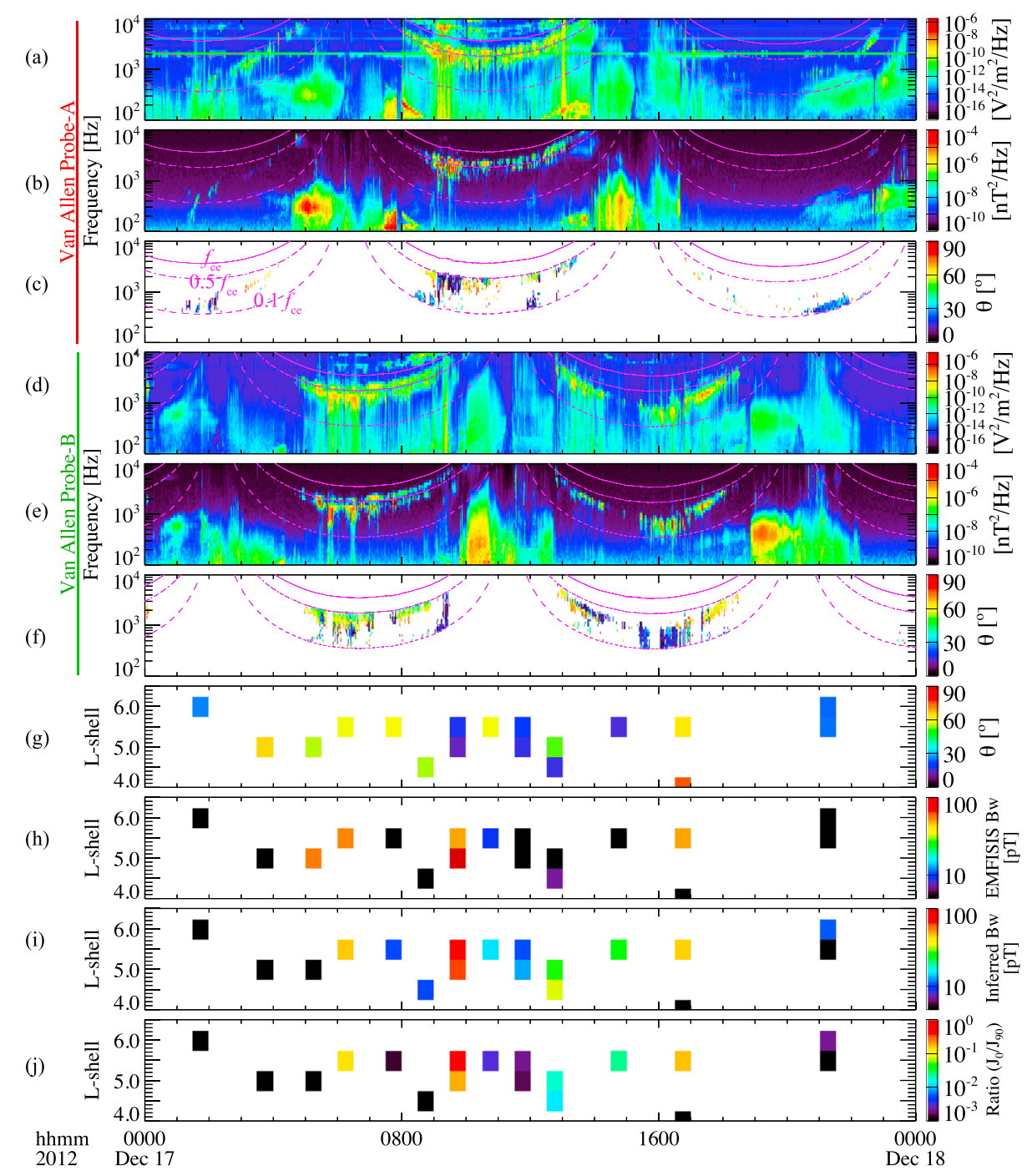

Figure 1. A comparison of whistler-mode waves observed by the EMFISIS instrument and their wave amplitudes inferred from the POES measurements. (a)-(c) Frequency-time spectrogram of electric field and magnetic field spectral density, and wave normal angles observed by Van Allen Probe A. Here the solid, dot-dashed, and dashed magenta lines indicate $f_{\text {ce, }}$ $0.5 f_{\mathrm{ce}}$, and $0.1 f_{\mathrm{ce}}$, where $f_{\mathrm{ce}}$ is the electron cyclotron frequency. (d)-(f) The same as Figures 1a-1c but observed by Van Allen Probe B. (g) Wave normal angles and (h) lower-band wave amplitudes integrated over $0.1-0.5 f_{\text {ce }}$ observed by two Van Allen Probes during the conjunction events with POES satellites. (i) Inferred whistler-mode wave amplitudes from POES electron measurements over $30-100 \mathrm{keV}$ and (j) ratios of precipitated and trapped electron fluxes over 30-100 keV during the conjunction events.

calculated in each bin for the measured and inferred waves separately (Figures $1 \mathrm{~h}$ and $1 \mathrm{i})$. We define a rough conjunction event if measurements from both Van Allen Probes and POES exist in the same bin, and these rough conjunction events are shown with colored bins in Figures $1 \mathrm{~g}-1 \mathrm{j}$. For each identified rough conjunction event, we calculate the median value of wave normal angles in each corresponding bin, which is shown in Figure 1g. For the conjunction events, in which relatively weak but very oblique waves were observed (e.g., 07:30-08:00 UT, 08:30-09:00 UT, and 10:30-11:00 UT), inferred wave amplitudes appear to be generally larger than those directly measured by the EMFISIS instrument, as shown in Figures $1 \mathrm{~g}-1 \mathrm{i}$. We note that since these are rough conjunction events, in which both measured and inferred wave amplitudes were averaged over a bin size of $0.5 \mathrm{~h}(\mathrm{UT}) \times 0.5 \mathrm{~L} \times 0.5 \mathrm{~h}(\mathrm{MLT})$, and there also exist uncertainties from the magnetic field mapping, the inferred wave amplitudes could deviate from the measured wave amplitudes (e.g., 05:00-05:30 UT and 14:30-15:00 UT). Nevertheless, in general the comparison of directly measured (Figure 1h) and inferred lower-band wave amplitudes (Figure 1i) exhibits fairly good agreement. 

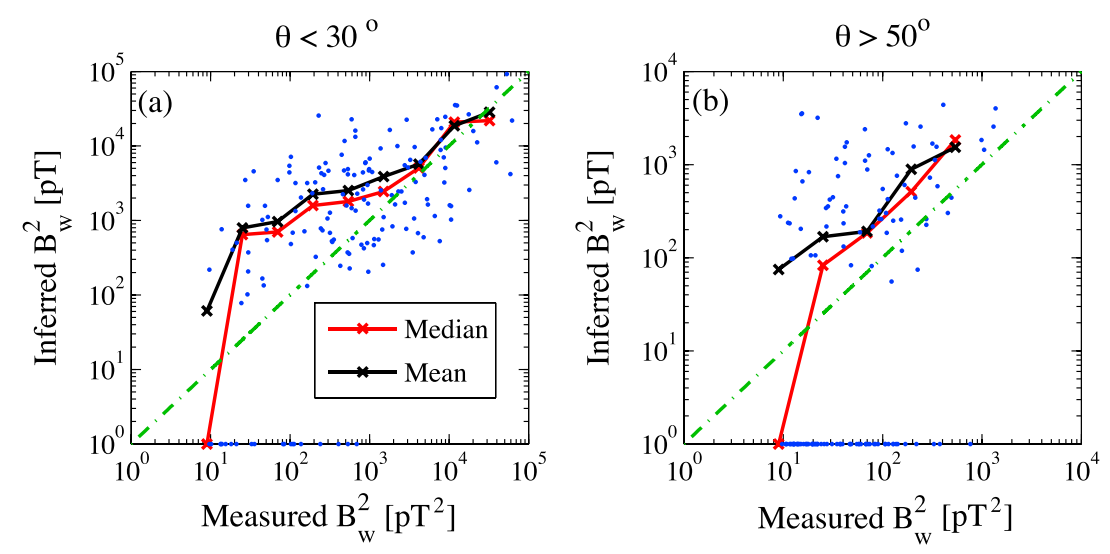

Figure 2. (a) Correlation of measured and inferred whistler-mode wave power $\left(B_{\mathrm{w}}{ }^{2}\right)$ for quasi-parallel waves $\left(\theta<30^{\circ}\right)$, where each blue dot represents one rough conjunction event, and the red (black) line indicates the median (mean) values. (b) The same as Figure 2a but for oblique waves $\left(\theta>50^{\circ}\right)$. The dash-dotted green line represents a ratio of inferred and measured wave power equal to 1 .

We apply this technique to hundreds of conjunction events between Van Allen Probes and POES satellites during the period from 1 October 2012 to 1 July 2013 and categorize each event into either quasi-parallel $\left(\theta<30^{\circ}\right)$ or oblique $\left(\theta>50^{\circ}\right)$ whistler-mode wave events, based on the recorded median value of wave normal angles. We identified 160 (139) conjunction events for quasi-parallel waves (oblique waves), all of which were collected from the region $4<L<6$ over 20-09 MLT. We excluded events where the background magnetic field measured by the Van Allen Probes deviates more than $25 \%$ from the dipole magnetic field to ensure that our identification of conjunction events using similar ranges in $L$-shell and MLT is approximately correct. This procedure also eliminated strongly disturbed periods, during which the effect of a non-dipolar magnetic field could be significant. However, this should not affect our essential conclusions in this study, where we focus on evaluating the differences in pitch angle scattering caused by quasi-parallel and oblique waves. Figure 2 shows two scatter plots of measured and inferred wave power $\left(B_{\mathrm{w}}{ }^{2}\right)$ from the conjunction events between Van Allen Probes and POES for the quasi-parallel (left) and oblique wave events (right), respectively. We categorized measured $B_{\mathrm{w}}{ }^{2}$ into 10 bins, which are logarithmically spaced over the range of $\sim 10-10^{5} \mathrm{pT}^{2}$, and calculated the mean (black crosses) and median values (red crosses) of the inferred $B_{\mathrm{w}}{ }^{2}$ in each measured $B_{\mathrm{w}}{ }^{2}$ bin. Note that a portion of inferred $B_{\mathrm{w}}{ }^{2}$ is set to $1 \mathrm{pT}^{2}$, since the precipitated electron fluxes during these events are below the background level, as discussed above. This means that the waves driving electron pitch angle scattering are too weak to lead to a significant electron precipitation that can be measured by the $0^{\circ}$ POES particle detector. Although this weak wave power could be $0.1 \mathrm{pT}^{2}$, $1 \mathrm{pT}^{2}$, or some other small values, setting it to the same small value of $\sim 1 \mathrm{pT}^{2}$ does not significantly affect the calculation of the median or mean values, which are shown in Figure 2, due to its small contribution. Therefore, these $B_{\mathrm{w}}{ }^{2} \sim 1 \mathrm{pT}^{2}$ were taken into account in the calculation of mean and median values of $B_{\mathrm{w}}{ }^{2}$ in order not to overestimate the result by artificially excluding these points with weak wave power. For quasiparallel wave events, the inferred values of $B_{\mathrm{w}}{ }^{2}$ are larger than the measured $B_{\mathrm{w}}{ }^{2}$ at low measured wave power $\left(B_{\mathrm{w}}{ }^{2}<\sim 400 \mathrm{pT}^{2}\right)$, whereas they agree quite well for higher $B_{\mathrm{w}}{ }^{2}$, with collected conjunction events close to the dash-dotted green line where the measured and inferred $B_{\mathrm{w}}{ }^{2}$ are identical. The fact that the mean and median values of inferred $B_{\mathrm{w}}{ }^{2}$ roughly coincide indicates an absence of significant experimental bias. However, for oblique wave events, the inferred $B_{\mathrm{w}}{ }^{2}$ is generally larger than the measured $B_{\mathrm{w}}{ }^{2}$ by a factor of $\sim 3$. We discuss the possible causes of this discrepancy in sections 3 and 4 .

\section{Numerical Calculation Results}

Electron pitch angle diffusion coefficients by lower-band whistler-mode waves are calculated by a full diffusion code [e.g., Artemyev et al., 2013a, 2013b], based on the method described by Glauert and Horne [2005] and Albert [2012]. Here we include -5 to +5 cyclotron harmonic resonances and the Landau resonance. Note that a dipole magnetic field model was used to calculate diffusion coefficients for simplicity, since it works well in the inner magnetosphere (4-6 $R_{\mathrm{E}}$ ) during not-too-disturbed periods. The plasmatrough density model 
(a)

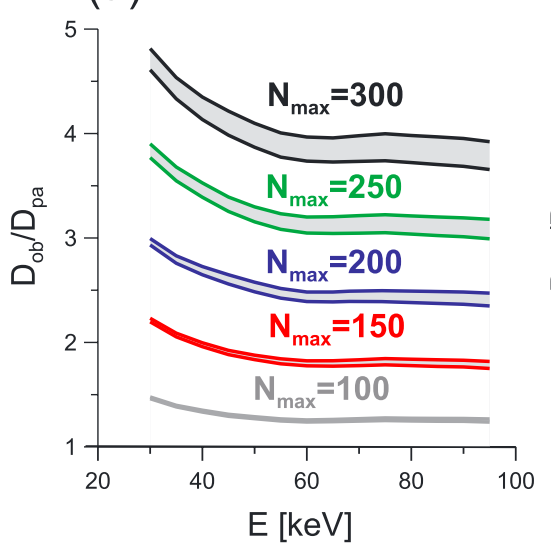

(b)

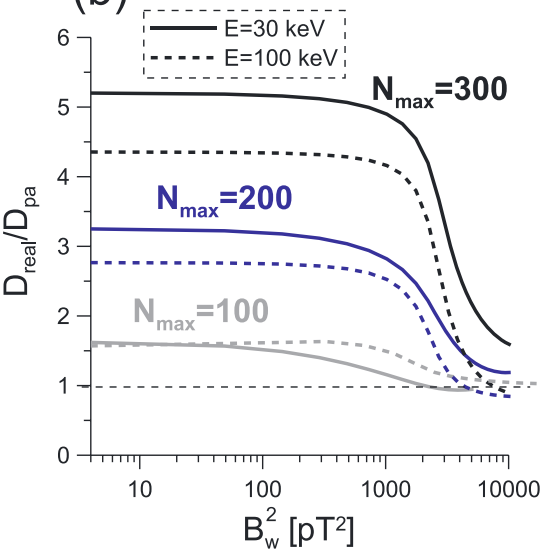

Figure 3. (a) Ratio between oblique and quasi-parallel diffusion rates $D_{\mathrm{ob}} / D_{\mathrm{pa}}$ as a function of energy at $L \sim 5$ with $\omega_{\mathrm{peo}} /$ $\Omega_{\mathrm{ce} 0}=4.7$. This ratio was calculated for the magnetic latitude range $|\lambda|<40^{\circ}$ for various values of $N_{\max }$ (color coded). The shaded region contained within solid lines of the same colors corresponds to loss cone angle values for $L=4.5$ to 5.5 . (b) Ratio of $D_{\text {real }} / D_{\text {pa }}$ as a function of whistler-mode wave power $\left(B_{\mathrm{w}}{ }^{2}\right)$ for an electron energy of 30 (solid lines) and $100 \mathrm{keV}$ (dashed lines). Different colors represent various values of $N_{\max }$, which are used to calculate the corresponding ratio.

from Sheeley et al. [2001] averaged over 20-09 MLT was used to calculate the ratio of angular plasma frequency $\left(\omega_{\mathrm{pe} 0}\right)$ to angular equatorial electron gyrofrequency $\left(\Omega_{\mathrm{ce} 0}\right)$ at the equator at $L \sim 5$, since whistler-mode waves analyzed in the conjunction events were located from the premidnight to the postdawn sector. The plasma density in the plasmatrough region is assumed to increase along the field lines with magnetic latitudes as

$$
n_{\mathrm{e}}(\lambda)=n_{\mathrm{e} 0} /(\cos \lambda)^{5},
$$

where $n_{\mathrm{e}}$ is the local plasma density at magnetic latitude $\lambda$ and $n_{\mathrm{e} 0}$ is the equatorial value [Denton et al., 2006]. We assume that the whistler-mode wave power spectral density follows a Gaussian frequency distribution with the peak frequency $\left(\omega_{m}\right)$ of $0.35 \Omega_{\text {ce0 }}$, lower and upper cutoffs of 0.125 and $0.5 \Omega_{\text {ceo }}$ and a bandwidth $\delta \omega=0.15 \Omega_{\text {ce0 }}$, where $\omega$ is the angular wave frequency.

We then calculate pitch angle diffusion coefficients for two groups of waves, quasi-parallel waves and very oblique waves, respectively. Wave amplitudes are assumed to remain constant along magnetic field lines from the equator to $\lambda \sim 40^{\circ}$ in rough agreement with Cluster statistics in the considered MLT domain during moderately disturbed periods of $D_{s t}>-25 \mathrm{nT}$ [Mourenas et al., 2014]. Wave normal angle distributions $g_{p a}(\theta)=\exp \left(-\left(\theta / \theta_{w, p a}\right)^{2}\right)$ and $g_{o b}(\theta)=\exp \left(-\left(\theta-\theta_{m}\right)^{2} /\left(\theta_{w, o b}\right)^{2}\right)$ are adopted for quasi-parallel and oblique waves, respectively, where $\theta_{w, p a}=30^{\circ}, \theta_{w, o b}=15^{\circ}$, and $\theta_{m}=70^{\circ}$. Bounce-averaged pitch angle diffusion coefficients $\left\langle D_{\alpha \alpha}\right\rangle$ are calculated at the bounce loss cone to evaluate the low-altitude electron precipitation measured by the POES satellites. We performed two sets of calculations for pitch angle scattering rates, yielding (1) the ratio $D_{\mathrm{ob}} / D_{\mathrm{pa}}$ and (2) the ratio $D_{\text {real }} / D_{\mathrm{pa}}$, where $D_{\mathrm{ob}}, D_{\mathrm{pa}}$ and $D_{\text {real }}$ represent bounce-averaged pitch angle diffusion coefficients at the bounce loss cone for oblique, quasi-parallel, and realistic whistlermode waves. A detailed definition of the "realistic" whistler-mode waves is given below.

Figure 3a shows the ratio of $D_{\mathrm{ob}} / D_{\mathrm{pa}}$ as a function of electron energy at $L \sim 5$ with $\omega_{\mathrm{pe} 0} / \Omega_{\mathrm{ce} 0}=4.7$ at the equator over the magnetic latitudes of $|\lambda|<40^{\circ}$. This ratio was calculated for various values of the upper bound on the refractive index $\left(N_{\max }\right)$, which is used to represent how close to the resonance cone the waves can be, and the diffusion coefficient is discarded at the point where the corresponding refractive index is above $N_{\text {max. }}$. It is important to note that for the very oblique waves close to the resonance cone the presence of $N_{\max }$ is expected as a result of a combination of thermal effects and Landau damping by suprathermal electrons near the resonance cone [e.g., Horne and Sazhin, 1990], which is discussed in more detail in section 4. Here the same magnetic wave amplitude is used to calculate $D_{\mathrm{ob}}, D_{\mathrm{pa}}$ and their ratio. A detailed examination of diffusion coefficients for 30-100 keV electrons shows that most electron scattering by oblique (quasi-parallel) waves occurs at $|\lambda| \sim 25^{\circ}-35^{\circ}\left(|\lambda|<15^{\circ}\right)$ (not shown). Therefore, $|\lambda|<40^{\circ}$ is reasonable to evaluate $D_{\mathrm{ob}} / D_{\mathrm{pa}}$, since this latitudinal range includes the most efficient scattering region for both quasi-parallel and oblique waves. $D_{\mathrm{ob}} / D_{\mathrm{pa}}$ is close to 1.5 for $N_{\max }=100$, is larger than 2 for $N_{\max }=150$, 
and becomes even larger for higher values of $N_{\max }=200$ to 300 . We note that the ratio $D_{\mathrm{ob}} / D_{\mathrm{pa}}$ is almost independent of electron energy in the considered range (30-100 keV) when almost all resonances for oblique and parallel waves are taken into account. Such features are consistent with rough analytical estimates [e.g., Mourenas et al., 2012].

In the case of realistic whistler-mode waves, we also use a constant average wave amplitude $B_{\mathrm{w}}$, which is roughly consistent with Cluster observations during quiet to moderately disturbed periods [e.g., Mourenas et al., 2014], while the distribution $g(\theta)$ depends on $B_{\mathrm{w}}$ and $\lambda$. The wave normal angle distribution $g(\theta)$ is obtained by approximating lower-band wave normal distributions from the Cluster statistics with polynomial fits. We consider a double distribution $g(\theta)=g_{\mathrm{pa}}+A\left(B_{\mathrm{w}}, \lambda\right) g_{\mathrm{ob}}$, where $g_{\mathrm{pa}}$ and $g_{\mathrm{ob}}$ are the same as above, while the coefficient $A\left(B_{\mathrm{w}}, \lambda\right)$ is derived by fitting Cluster mean values (detailed values used for calculations are shown in the supporting information). Cluster statistics shows that the amount of very oblique waves increases as $|\lambda|$ increases, while it decreases as $B_{\mathrm{w}}$ increases [e.g., Mourenas et al., 2014]. Such an evolution is probably due to the refraction of whistler-mode waves to progressively larger wave normal angles as they propagate along the field lines to higher latitudes [e.g., Horne and Thorne, 2003]. Figure $3 \mathrm{~b}$ shows the ratio of $D_{\text {real }} / D_{\text {pa }}$ as a function of wave power for $30 \mathrm{keV}$ (solid lines) and $100 \mathrm{keV}$ (dashed lines) over $|\lambda|<40^{\circ}$. For $N_{\max } \geq 100$ this ratio is larger than 1.5 for small $B_{\mathrm{w}}{ }^{2}<\sim 400 \mathrm{pT}^{2}$, while it decreases to $\sim 1$ for $B_{\mathrm{w}}{ }^{2}>\sim 4000 \mathrm{pT}^{2}$. This ratio also becomes larger as $N_{\max }$ increases due to the increased contribution of higher-order cyclotron harmonic resonances. The trend of $D_{\text {real }} / D_{\text {pa }}$ is similar for both 30 and $100 \mathrm{keV}$ electrons, although the values are slightly larger at $30 \mathrm{keV}$ compared to $100 \mathrm{keV}$.

\section{Interpretation of Observations and Computation Results}

For very oblique whistler-mode waves near the resonance cone, the phase speed of the waves approaches the speed of thermal electrons, and thermal effects become important, as well as the Landau damping by hot electrons. Nevertheless, the cold-plasma refractive index, which steeply increases near the resonance cone angle, should be only slightly modified by thermal effects as long as it remains smaller than the value of the quasi-electrostatic expression of the refractive index [e.g., Horne and Sazhin, 1990; Hashimoto et al., 1977]. Furthermore, very oblique waves can also be damped by the Landau resonance. By considering these two effects, $N_{\max }$ can be estimated using the given plasma parameters. During quiet to moderately disturbed periods, the calculated $N_{\max }$ is in the range of 130-280 (with larger values for more quiet periods) over $|\lambda| \sim 25^{\circ}-35^{\circ}$, where pitch angle scattering of $30-100 \mathrm{keV}$ electrons by oblique waves is most efficient. More details on the estimation of $N_{\max }$ are provided in the supporting information.

First, we discuss the conjunction events between POES and Van Allen Probes during which only oblique waves were observed by Van Allen Probes. As shown in Figure $2 b$, the mean ratio of inferred and measured wave power is $\sim 3$ for measured wave power $B_{w}{ }^{2}>25 \mathrm{pT}^{2}$. Since wave power is proportional to pitch angle diffusion coefficients [e.g., Glauert and Horne, 2005], the ratio of inferred and measured wave power is equivalent to the ratio of pitch angle diffusion coefficients inferred from the POES electron measurements and calculated using wave amplitudes directly measured by the Van Allen Probes under the assumption that the waves are quasi-parallel. Therefore, the ratio $(\sim 3)$ of inferred and measured wave power agrees well with $D_{\mathrm{ob}} / D_{\mathrm{pa}} \sim 2-2.5$ for $N_{\max } \sim 150$, which should correspond to moderately disturbed periods. However, for periods of weaker oblique wave power $B_{\mathrm{w}}{ }^{2}<25 \mathrm{pT}^{2}$, which are representative of very oblique whistler-mode wave amplitudes obtained onboard Cluster during quiet times [Agapitov et al., 2013], we might need to use the maximum, quiet time upper-bounds $N_{\max } \sim 200-300$ at high latitudes to estimate $D_{\mathrm{ob}} / D_{\text {pa }}$. As shown in Figure $2 \mathrm{~b}$, for weaker oblique waves, the ratio between the inferred and measured wave power becomes larger ( 6), which is roughly consistent with the larger ratio at $N_{\max } \sim 300$ shown in Figure 3a.

It is important to mention that during conjunction events in which mainly quasi-parallel waves were recorded by Van Allen Probes, oblique waves were not necessarily absent at higher latitudes, since the Van Allen Probe wave observation is mostly limited within $20^{\circ}$ of the magnetic equator. The wave normal angles of initially quasi-parallel waves tend to become larger as they propagate to higher latitudes [Thorne and Kennel, 1967; Chen et al., 2013], and the probability of observing oblique waves increases at higher magnetic latitudes, as shown in Cluster statistics [Agapitov et al., 2013; Artemyev et al., 2013a]. Therefore, in order to use a more realistic wave normal distribution as a function of magnetic latitude, we performed numerical fits to the mean wave-normal distribution $g(\theta, \lambda)$ as a function of RMS wave amplitude $B_{\mathrm{w}}$ obtained from the Cluster data over 
L-shells of 4-6. It is more reasonable to compare such fits with the mean ratios of scattering rates derived from Van Allen Probes and POES, when quasi-parallel waves are mainly observed near the magnetic equator.

Using these fits, the ratio $D_{\text {real }} / D_{\text {pa }}$ is calculated numerically for electrons with energies of 30 and $100 \mathrm{keV}$, respectively, as shown in Figure $3 \mathrm{~b}$. Considering nearly quiet time values for $N_{\max } \sim 200-300$ at high latitudes, this ratio can reach a value of about 3-5 for small wave power $B_{\mathrm{w}}{ }^{2} \sim 4$ to $400 \mathrm{pT}^{2}$, which are representative of quiet to moderately disturbed geomagnetic conditions [Artemyev et al., 2013a]. Even larger $D_{\text {real }} / D_{\text {pa }}$ would be obtained for smaller $\omega_{\mathrm{pe} 0} / \Omega_{\mathrm{ce} 0}$. For higher full wave power $B_{\mathrm{w}}{ }^{2}>4000 \mathrm{pT}^{2}$, the same ratio is reduced to about 1 due to the near-suppression of very oblique waves at high latitudes. This trend is consistent with Figure $2 \mathrm{a}$, in which quasi-parallel waves were predominantly observed within $20^{\circ}$ of the magnetic equator.

\section{Summary and Discussion}

We analyze conjunction events between the Van Allen Probes located in the magnetosphere near the geomagnetic equator and the polar-orbiting POES satellites located at conjugate low altitudes to evaluate electron pitch angle scattering rates driven by quasi-parallel and very oblique whistler-mode waves, respectively. We found that the mean wave amplitudes inferred from POES electron measurements under the assumption of quasi-parallel wave propagation are significantly larger than those directly measured by Van Allen Probes for the conjunction events that include mostly oblique waves or quasi-parallel waves with small amplitudes $\left(B_{\mathrm{w}}<\sim 20 \mathrm{pT}\right)$, whereas the measured and inferred wave amplitudes are generally consistent for mostly quasi-parallel wave events with relatively strong wave amplitudes $\left(B_{\mathrm{w}}>\sim 20 \mathrm{pT}\right)$. The comparisons between the observational and numerical calculation results suggest that pitch angle scattering rates derived from direct wave measurements of Van Allen Probes and inferred from the twodirectional POES electron measurements during rough conjunction events provide plausible experimental evidence of the potentially important role that very oblique waves can play in scattering electrons towards the loss cone. Very oblique waves with the same magnetic wave amplitudes as the quasi-parallel waves can considerably increase pitch angle scattering rates near the loss cone, which is consistent with previous studies [Artemyev et al., 2012, 2013b; Mourenas et al., 2012].

Acknowledgments

This work was supported by JHU/APL contracts 967399 and 921647 under NASA's prime contract NAS5-01072 and the NASA LWS TR\&T program. The analysis at UCLA was supported by the EMFISIS sub-award 1001057397:01, ECT sub-award 13-041, NASA grants NNX11AD75G, NNX11AR64G, NNX12AD12G, and NNX13AI61G, and NSF GEM grant of AGS-1405054. Work of A. Artemyev was partially supported by the Russian Ministry of Education (project \# MK-1781.2014.2). J. Albert would like to acknowledge NASA grant NNH11AR78I. B. Ni thanks the support from the NSFC grant 41204120 and from the Fundamental Research Funds for the Central Universities grant 2042014kf0251. We acknowledge the Van Allen Probes data from the EMFISIS instrument obtained from https://emfisis. physics.uiowa.edu/data/index. We also would like to thank the NOAA POES team especially Janet Green for providing the NOAA POES data (obtained from http://satdat.ngdc.noaa.gov/sem/poes/ data/) and helpful advice on the use of the data. We are also grateful for the ESA Cluster Active Archive (http://caa.estec. esa.int/caa/home.xml) for providing the STAFF-SA dataset.

The Editor thanks two anonymous reviewers for their assistance in evaluating this paper.
The present study also emphasizes the importance of thermal effects and the Landau damping caused by hot electrons when treating very oblique whistler-mode waves near the resonance cone. In particular, the natural variability of the thermal and suprathermal electron populations in the outer belt dependent on geomagnetic activity could explain the factor of $\sim 3-5$ variation around the mean value of scattering rates obtained on the basis of POES measurements, particularly for small wave amplitudes. Therefore, it will be important to conduct more accurate warm plasma calculations of the refractive index based on the electron populations observed by the Van Allen Probes, but this is beyond the scope of the present paper and is left for a future study.

\section{References}

Agapitov, O., A. Artemyev, V. Krasnoselskikh, Y. V. Khotyaintsev, D. Mourenas, H. Breuillard, M. Balikhin, and G. Rolland (2013), Statistics of whistler-mode waves in the outer radiation belt: Cluster STAFF-SA measurements, J. Geophys. Res. Space Physics, 118, 3407-3420, doi:10.1002/jgra.50312.

Albert, J. M. (2012), Dependence of quasi-linear diffusion coefficients on wave parameters, J. Geophys. Res., 117, A09224, doi:10.1029/ 2012JA017718.

Artemyev, A. V., O. V. Agapitov, D. Mourenas, V. Krasnoselskikh, and L. M. Zelenyi (2013a), Storm-induced energization of radiation belt electrons: Effect of wave obliquity, Geophys. Res. Lett., 40, 4138-4143, doi:10.1002/grl.50837.

Artemyev, A. V., D. Mourenas, O. V. Agapitov, and V. V. Krasnoselskikh (2013b), Parametric validations of analytical lifetime estimates for radiation belt electron diffusion by whistler waves, Ann. Geophys., 31, 599-624, doi:10.5194/angeo-31-599-2013.

Artemyev, A., O. Agapitov, H. Breuillard, V. Krasnoselskikh, and G. Rolland (2012), Electron pitch-angle diffusion in radiation belts: The effects of whistler wave oblique propagation, Geophys. Res. Lett., 39, L08105, doi:10.1029/2012GL051393.

Breuillard, H., Y. Zaliznyak, V. Krasnoselskikh, O. Agapitov, A. Artemyev, and G. Rolland (2012), Chorus wave-normal statistics in the Earth's radiation belts from ray tracing technique, Ann. Geophys., 30, 1223-1233, doi:10.5194/angeo-30-1223-2012.

Burton, R. K., and R. E. Holzer (1974), The origin and propagation of chorus in the outer magnetosphere, J. Geophys. Res., 79(7), 1014-1023, doi:10.1029/JA079i007p01014.

Chen, L., R. M. Thorne, W. Li, and J. Bortnik (2013), Modeling the wave normal distribution of chorus waves, J. Geophys. Res. Space Physics, 118, 1074-1088, doi:10.1029/2012JA018343.

Denton, R. E., K. Takahashi, I. A. Galkin, P. A. Nsumei, X. Huang, B. W. Reinisch, R. R. Anderson, M. K. Sleeper, and W. J. Hughes (2006), Distribution of density along magnetospheric field lines, J. Geophys. Res., 111, A04213, doi:10.1029/2005JA011414.

Evans, D. S., and M. S. Greer (2004), Polar Orbiting Environmental Satellite Space Environment Monitor-2: Instrument descriptions and archive data documentation, NOAA Tech. Mem. 93, version 1.4, Space Weather Predict. Cent., Boulder, Colo. 
Glauert, S. A., and R. B. Horne (2005), Calculation of pitch angle and energy diffusion coefficients with the PADIE code, J. Geophys. Res., 110, A04206, doi:10.1029/2004JA010851.

Goldstein, B. E., and B. T. Tsurutani (1984), Wave normal directions of chorus near the equatorial source region, J. Geophys. Res., 89(A5), 2789-2810, doi:10.1029/JA089iA05p02789.

Green, J. C. (2013), MEPED telescope data processing algorithm theoretical basis document, Natl. Oceanic and Atmos. Admin. Space Environ. Cent., Boulder, Colo.

Hashimoto, K., I. Kimura, and H. Kumagai (1977), Estimation of electron temperature by VLF waves propagating in directions near the resonance cone, Planet. Space Sci., 25, 871-877.

Hayakawa, M., Y. Yamanaka, M. Parrot, and F. Lefeuvre (1984), The wave normals of magnetospheric chorus emissions observed on board GEOS 2, J. Geophys. Res., 89(A5), 2811-2821, doi:10.1029/JA089iA05p02811.

Horne, R. B., and S. S. Sazhin (1990), Quasielectrostatic and electrostatic approximations for whistler mode waves in the magnetospheric plasma, Planet. Space Sci., 38, 311-318.

Horne, R. B., and R. M. Thorne (2003), Relativistic electron acceleration and precipitation during resonant interactions with whistler-mode chorus, Geophys. Res., Lett., 30(10), 1527, doi:10.1029/2003GL016973.

Kletzing, C. A., et al. (2013), The Electric and Magnetic Field Instrument Suite and Integrated Science (EMFISIS) on RBSP, Space Sci. Rev., doi:10.1007/s11214-013-9993-6.

Lam, M. M., R. B. Horne, N. P. Meredith, S. A. Glauert, T. Moffat-Griffin, and J. C. Green (2010), Origin of energetic electron precipitation $>30 \mathrm{keV}$ into the atmosphere, J. Geophys. Res., 115, A00F08, doi:10.1029/2009JA014619.

Lauben, D. S., U. S. Inan, T. F. Bell, and D. A. Gurnett (2002), Source characteristics of ELF/VLF chorus, J. Geophys. Res., 107(A12), 1429, doi:10.1029/2000JA003019.

Li, W., R. M. Thorne, J. Bortnik, Y. Nishimura, V. Angelopoulos, L. Chen, J. P. McFadden, and J. W. Bonnell (2010a), Global distributions of suprathermal electrons observed on THEMIS and potential mechanisms for access into the plasmasphere, J. Geophys. Res., 115, A00J10, doi:10.1029/2010JA015687.

Li, W., et al. (2010b), THEMIS analysis of observed equatorial electron distributions responsible for the chorus excitation, J. Geophys. Res., 115, A0OF11, doi:10.1029/2009JA014845.

Li, W., R. M. Thorne, J. Bortnik, Y. Y. Shprits, Y. Nishimura, V. Angelopoulos, C. Chaston, O. Le Contel, and J. W. Bonnell (2011), Typical properties of rising and falling tone chorus waves, Geophys. Res. Lett., 38, L14103, doi:10.1029/2011GL047925.

Li, W., J. Bortnik, R. M. Thorne, C. M. Cully, L. Chen, V. Angelopoulos, Y. Nishimura, J. B. Tao, J. W. Bonnell, and O. LeContel (2013a), Characteristics of the Poynting flux and wave normal vectors of whistler-mode waves observed on THEMIS, J. Geophys. Res. Space Physics, 118, 1461-1471, doi:10.1002/jgra.50176.

Li, W., B. Ni, R. M. Thorne, J. Bortnik, J. C. Green, C. A. Kletzing, W. S. Kurth, and G. B. Hospodarsky (2013b), Constructing the global distribution of chorus wave intensity using measurements of electrons by the POES satellites and waves by the Van Allen Probes, Geophys. Res. Lett., 40, 4526-4532, doi:10.1002/grl.50920.

Mauk, B. H., N. J. Fox, S. G. Kanekal, R. L. Kessel, D. G. Sibeck, and A. Ukhorskiy (2012), Science objectives and rationale for the Radiation Belt Storm Probes Mission, Space Sci. Rev., 1-15, doi:10.1007/s11214-012-9908-y.

Mourenas, D., A. V. Artemyev, J.-F. Ripoll, O. V. Agapitov, and V. V. Krasnoselskikh (2012), Timescales for electron quasi-linear diffusion by parallel and oblique lower-band chorus waves, J. Geophys. Res., 117, A06234, doi:10.1029/2012JA017717.

Mourenas, D., A. V. Artemyev, O. V. Agapitov, and V. V. Krasnoselskikh (2014), Consequences of geomagnetic activity on energization and loss of radiation belt electrons by oblique chorus waves, J. Geophys. Res. Space Physics, 119, 2775-2796, doi:10.1002/2013JA019674.

$\mathrm{Ni}$, B., R. M. Thorne, Y. Y. Shprits, and J. Bortnik (2008), Resonant scattering of plasma sheet electrons by whistler-mode chorus: Contribution to diffuse auroral precipitation, Geophys. Res. Lett., 35, L11106, doi:10.1029/2008GL034032.

Ni, B., W. Li, R. M. Thorne, J. Bortnik, J. C. Green, C. A. Kletzing, W. S. Kurth, G. B. Hospodarsky, and M. de Soria-Santacruz Pich (2014), A novel technique to construct the global distribution of whistler mode chorus wave intensity using low-altitude POES electron data, J. Geophys. Res. Space Physics, 119, 5685-5699, doi:10.1002/2014JA019935.

Santolík, O., M. Parrot, and F. Lefeuvre (2003), Singular value decomposition methods for wave propagation analysis, Radio Sci., 38(1), 1010, doi:10.1029/2000RS002523.

Santolík, O., D. A. Gurnett, J. S. Pickett, J. Chum, and N. Cornilleau-Wehrlin (2009), Oblique propagation of whistler mode waves in the chorus source region, J. Geophys. Res., 114, A00F03, doi:10.1029/2009JA014586.

Santolík, O., E. Macúšová, I. Kolmašová, N. Cornilleau-Wehrlin, and Y. de Conchy (2014), Propagation of lower-band whistler-mode waves in the outer Van Allen belt: Systematic analysis of 11 years of multi-component data from the Cluster spacecraft, Geophys. Res. Lett., 41, 2729-2737, doi:10.1002/2014GL059815.

Sheeley, B. W., M. B. Moldwin, H. K. Rassoul, and R. R. Anderson (2001), An empirical plasmasphere and trough density model: CRRES observations, J. Geophys. Res., 106(A11), 25,631-25,641, doi:10.1029/2000JA000286.

Shprits, Y. Y., and B. Ni (2009), Dependence of the quasi-linear scattering rates on the wave normal distribution of chorus waves, J. Geophys. Res. 114, A11205, doi:10.1029/2009JA014223.

Thorne, R. M., and C. F. Kennel (1967), Quasi-trapped VLF propagation in the outer magnetosphere, J. Geophys. Res., 72(3), 857-870, doi:10.1029/JZ072i003p00857.

Thorne, R. M., B. Ni, X. Tao, R. B. Horne, and N. P. Meredith (2010), Scattering by chorus waves as the dominant cause of diffuse auroral precipitation, Nature, 467, 943-946, doi:10.1038/nature09467.

Wilson, L. B. I. I. I., C. A. Cattell, P. J. Kellogg, J. R. Wygant, K. Goetz, A. Breneman, and K. Kersten (2011), The properties of large amplitude whistler mode waves in the magnetosphere: Propagation and relationship with geomagnetic activity, Geophys. Res. Lett., 38, L17107, doi:10.1029/2011GL048671. 